\title{
Effective Congestion Avoidance Scheme for Mobile Ad Hoc Networks
}

\author{
S.Sheeja \\ Research Scholar, Bharathiar University, Coimbatore. \\ sheejakhader@yahoo.com \\ Dr.Ramachandra.V.Pujeri, \\ Vice-Principal, KGiSL Institute of Technology, Saravanampatti, Coimbatore -641035, Tamil Nadu, INDIA \\ sriramu.vp@gmail.com
}

\begin{abstract}
Mobile nodes are organized randomly without any access point in Mobile Ad hoc Networks (MANETs). Due to the mobility of nodes, the network congestion occurs. So many congestion control mechanisms were proposed to avoid the congestion avoidance or reducing the congestion status. In this research work, we proposed to develop the Effective Congestion Avoidance Scheme (ECAS), which consists of congestion monitoring, effective routing establishment and congestionless based routing. The overall congestion status is measured in congestion monitoring. In routing establishment, we propose the contention metric in the particular channel in terms of, queue length of packet, overall congestion standard, packet loss rate and packet dropping ratio to monitor the congestion status. Based on the congestion standard, the congestionless based routing is established to reduce the packet loss, high overhead, long delay in the network. By extensive simulation, the proposed scheme achieves better throughput, packet delivery ratio, low end-to-end delay and overhead than the existing schemes.
\end{abstract}

Keywords - MANET, throughput, delivery ratio, packet loss, overall congestion standard, queue length, contention metric, flow control and congestion control

\section{INTRODUCTION}

\section{A. Mobile Ad Hoc Networks (MANET)}

Mobile ad-hoc network is a collection of mobile nodes forming an ad-hoc network without the assistance of any centralized structures. These networks introduced a new art of network establishment and can be well suited for an environment where either the infrastructure is lost or where deploy an infrastructure is not very cost effective. The popular IEEE 802.11 "WI-FI" protocol is capable of providing ad-hoc network facilities at low level, when no access point is available. However in this case, the nodes are limited to send and receive information but do not route anything across the network. Mobile ad-hoc networks can operate in a standalone fashion or could possibly be connected to a larger network such as the Internet. Mobile ad-hoc networks can turn the dream of getting connected "anywhere and at any time" into reality.

\section{B. Congestion Control in MANETs}

Congestion takes place in MANETs with limited resources. In these networks, shared wireless channel and dynamic topology leads to interference and fading during packet transmission. Packet victims and bandwidth dilapidation are caused due to congestion, and thus, time and energy is wasted during its recovery. Congestion can be prevented using congestion-aware protocol through bypassing the affected links [16]. Severe throughput degradation and massive fairness problems are some of the identified congestion related problems. These problems are generated from MAC, and protocol routing and transport layers.

Congestion control is the main problem in ad-hoc networks. Congestion control is associated to controlling traffic incoming into a telecommunication network. To avoid congestive crumple or link capabilities of the intermediate nodes and networks and to reduce the rate of sending packets congestion control is used extensively [17]. Congestion control and dependability mechanisms are combined by TCP to perform the congestion control without explicit feedback about the congestion position and without the intermediate nodes being directly intermittent [17].

Their principles include packet conservation, additive increase and multiplicative decrease in sending rate, stable network. End system flow control, network congestion control, network based congestion avoidance, and resource allotment includes the basic techniques for congestion control [18].

Packet failure in MANETs is primarily caused due to obstruction. Involving congestion control over a mobility and failure adaptive routing protocol at the network layer can condense the packet loss. The congestion nonadaptive routing protocols, leads to the following difficulties:

Extensive delay: Most of the congestion control mechanism takes much time for detecting congestion. Sometimes the usage of new routes in some critical situations is advisable. The main problem is the delay stirring for route searching in on-demand routing protocol.

More Overhead: Congestion control mechanism takes effort for processing and communication in new routes for discovering it. It also takes effort in multipath routing 
for maintaining the multi-paths, though there is another protocol.

Heavy packet losses: Once the congestion is detected the packets may be lost. Congestion control solution is applied either by decreasing the sending rate at the sender, or dropping packets at the intermediate nodes or by both methods to decrease the traffic load. Due to high packet loss rate, small throughput may be occurred.

\section{RELATED WORKS}

V. Thilagavathe and Dr. K. Duraiswamy [3] proposed the cross-layer based technique to overcome congestion that occurs in MAC and transport layer in MANET. The proposed technique was applied over a Adhoc On demand Multipath Reliable and Energy Aware QoS Routing Protocol (AOMP-REQR). The technique of additive increase and multiplicative decrease (AIMD) was applied for rate based congestion control of transport layer protocol. If source receives congestion status information from both MAC and transport layer simultaneously for the same route, then congestion free route can be established for transmission, without performing rate control.

Karunakaran et.al [4] presented a Cluster Based Congestion Control (CBCC) protocol that consists of scalable and distributed cluster-based mechanisms for supporting congestion control in Adhoc networks. The distinctive feature of approach was that it was based on the self-organization of the network into clusters. The clusters autonomously and proactively monitor congestion within its localized scope. The protocol consists of clustering mechanism, traffic rate estimation and traffic rate adjustment. The present approach improved the responsiveness of the system when compared to end-to-end techniques. After estimating the traffic rate along a path, the sending rate of the source nodes was adjusted accordingly. Thus the protocol looked forward the injection of dynamic flows in the network and proactively adjusts the rate while waiting for congestion feedback.

Senthil kumaran et.al [6] presented the Congestion Free Routing in Ad hoc networks (CFR), based on dynamically estimated mechanism to monitor network congestion by calculating the average queue length at the node level. While using the average queue length, the nodes' congestion status divided into the three zones (safe zone, likely to be congested zone and congested zone). CFR utilized the non-congested neighbours and initiates route discovery mechanism to discover a congestion free route between source and destination. This path became a core path between source and destination. To maintain the congestion free status, the nodes, which were helping data packet transmission periodically, calculate their congestion status at the node level. When a core node was noticed that it fell in to likely to congested zone and alerts to its neighbours. The predecessor core path node was aware of this situation and initiates an alternate path discovery mechanism to a destination. Finally it discovered a new congestion free route to the destination.
Rajeswari et.al [7] presented a simulation-based performance evaluation and comparison of three queuing techniques for different number of nodes, packet size and pause time for the impact of using random-early drop as compared to drop-tail policy and weighted fair scheduling. The simulation results shown WFQ that outperforms other disciplines in terms of Throughput and packet delivery ratio although RED are also very close to it for the considered node scenarios. They also noticed that using RED has greatly improved all the performance measures especially with FIFO. The reason was that RED monitors the average queue size and randomly drops packets when congestion was detected.

Mohammad Amin Kheirandish Fard et.al [13] explored an end-to-end threshold-based algorithm, which enhances congestion control to address link failure loss in MANET. It consists of two phases. First, threshold-based loss classification algorithm distinguished losses due to link failure by estimating queue usage based on Relative Oneway Trip Time (ROTT). Second phase adjusted RTO for new route by comparing capabilities of new route to the broken route using available information in Transport layer such as ROTT and number of hops. Thresholdbased loss classification algorithm used queue usage to classify network state periodically into congestion or non-congestion mode. Any retransmission timeout in period, which MANET was non-congested mode, is an indication of link failure loss. In addition, implementation showed that small percentage of three duplicate acknowledgments that emerge immediately after route recovery might be result of route changes.

Kazuya et.al [14] analyzed a routing protocol that uses multi-agents to reduce network congestion for a Mobile Ad hoc NETwork (MANET). MANET is a multi-hop wireless network in which the network components such as PC, PDA and mobile phones are mobile. The components can communicate with each other without going through a server. Two kinds of agents are engaged in routing. One is a Routing Agent that collects information about network congestion as well as link failure. The other is a Message Agent that uses this information to get to their destination nodes.

Nazia Zaman et.al [15] explored a router assisted approach, where routers provide explicit feedback which allows quick increase of throughput. The router can provide feedback through which the sender node can distinguishes between congestion based packet loss and non-congestion based losses. In addition, to determine the type of loss, the sender also controls its sending rate using this feedback.

Claudio et.al [20] presented a reliable protocol that was deployed to cross layer congestion control where the video coding is aware. Here they proposed a congestion avoidance framework designed for multi-tree overlays in mobile ad-hoc environments with high node density and stringent constraints on delay.

Santhosh and Narasimhan [21] explored a hop by hop congestion aware routing protocol, which employs a combined weight values. They have considered the metrics like data rate, queuing delay, link quality and 
MAC overhead. The route is discovered with minimum cost index is selected which is based on the node weight of all the in-network nodes. Their proposed approach achieves high throughput, high packet delivery ratio, low packet drop and delay.

The paper is organized as follows. The Section 1 describes with overview of congestion and its issues. Section 2 deals with the Related Work. Section 3 is devoted for the implementation of proposed algorithm. Section 4 describes the performance analysis and the last section concludes the work.

\section{IMPLEMENTATION OF PROPOSED ALGORITHM}

Our proposed congestion control mechanism is based on mobility and the design is carried out in the Network Layer.

We proposed the concept of Congestion status; effective routing establishment based on congestion detection, establishing congestion less routing.

\section{A. Scanning the status of Congestion}

If the number of packets coming to a particular mobile node exceeds its carrying capacity, the mobile node becomes congested and starts losing packets. Some of the metrics can be used for a mobile node to monitor congestion status. Cluster head among these mobile nodes are the percentage of all packets discarded for lack of buffer space, the average queue length, the number of packets timed out and retransmitted, the average packet delay, packet duplicated, packet and the standard deviation of packet delay. In all these cases, rising numbers indicate growing congestion. Here a node periodically checks the occupancy of its link-layer buffer. The congestion status is determined based on the ratio of packet dropping between the numbers of packets currently buffered to the buffer size.

\section{B. Effective Routing Establishment based on congestion detection}

For the establishment of effective routing of the packets, the congestion is defined. When queue length is near capacity or when battery level fell below threshold, the overall congestion can be detected. The Overall congestion standard is determined based on the channel activity, queue length, probability of queue and packet dropping ratio.

1. Avg_que $1=0$

2. Inst_que $1=0$

3. Minth $=0.45 *$ buffer_size

4. Maxth $=0.65 *$ buffer_size

5. Initialize the node configurations

6. Inst_que1++

//Calculate average queue length

The main goal is to acquire network statistics using a heuristic approach. The traffic rate is computed as follows: Let the value $\mathrm{L}_{\text {offk }}$ represent the offered load at the queue of node $\mathrm{k}$ and it is defined as

$$
L_{o f f k}=\frac{A_{v R}}{S_{R}}
$$

Where $A_{v R}$ is the aggregate arrival rate of the packets produced and forwarded at node $\mathrm{k}$ while $\mathrm{S}_{\mathrm{R}}$ is the service rate at node $\mathrm{k}$, i.e., $\mathrm{T} / \mathrm{SR}_{\mathrm{i}}=1$ where $\mathrm{T}$ is the computed exponentially weighted moving average of the packets' waiting time at the head of the service queue. The distribution of the queue length (essentially this is the probability that there are $1 \mathrm{Q}$ packets in the queue) at the node is computed as

$$
P(Q)=\left(1-L_{o f f k}\right) L_{o f f k}^{1}
$$

7. If the queue length is not empty

8. Avg_que $1=\left(1-\mathrm{w}_{\mathrm{q}}\right)$ Avg_que1+Inst_que1 $* \mathrm{w}_{\mathrm{q}}$

$\mathrm{w}_{\mathrm{q}}$ is weighted queue length of the window.

9. If ( Inst_que1 > Avg_que1)

10. Queue_status = Inst_que1 - Avg_que1

11. Else

12. Queue_status $=0$

13. If (Inst_que1> PDR)

14. If(Queue_status $>\operatorname{Min}_{\text {th }}$ and Inst_Que $<\operatorname{Max}_{\text {th }}$ )

15. Call procedure to Create Congestion monitoring

16. Else Queue is in congested area

17. For each departing packet in the queue

18. Inst_que1 - -

19. Determine the channel activity

The amount of time between the receipt of one packet and the transmission of the next is called a Short Inter Frame Space (SIFS). Channel activity is calculated from Request-To-Send (RTS), Clear-to-Send (CTS), data, acknowledgement (ACK) and Packet Loss Rate. Then the channel activity due to MAC contention will be

$$
C_{\text {act }}=t_{R T S}+t_{C T S}+4 t_{\text {SIFS }}+t_{\text {access }}+P_{L R}
$$

Where $t_{\text {RTS }}$ and $t_{\text {CTS }}$ are the time consumed on RTS and CTS, respectively and $t_{\text {SIFS }}$ is the SIFS period. $t_{\text {access }}$ is the time taken due to access contention. Packet Loss Rate is given by

$P_{L R}\left(t_{1}, t_{2}\right)=\frac{\int_{t_{1}}^{t_{2}} 1_{\left\{G(t)=D_{l}\right\}} d F(t)}{\int_{t_{1}}^{t_{2}} d F(t)}$

Where $\mathrm{F}(t)$ is the arrival process for user packets. Here, the denominator represents the number of user packets sent in $\left(t_{1}, t_{2}\right)$ and the numerator represents the number of lost user Packets. Overall Congestion standard is given as

Overall Congestion Standard $=\mathrm{C}_{\mathrm{act}}+$ Queue length $+\mathrm{P}(\mathrm{Q})$ + Packet Dropping Ratio (PDR)

The Packet Dropping Ratio (PDR) is calculated as 


$$
\text { PacketDroppingRatio }=\frac{P_{d n} * P_{m n}}{P_{t n}} * 100
$$

Where $\mathrm{P}_{\mathrm{dn}}=$ No of packets dropped

$\mathrm{P}_{\mathrm{mn}}=$ No. of packets misrouted

$\mathrm{P}_{\mathrm{tn}}=$ Total No of packets transmitted.

If the overall congestion standard is low, the routing is called as effective routing. The packets are routed with minimum number of congestion standard and minimum Drop tail value

\section{Establishing a congestion less routing}

Step 1: Source node $S$ selects the number of on hop neighbours and replicates the mobile nodes to the particular neighbours.

Step 2: The shortest path is determined by the mobile node to move towards the intermediate nodes.

Step 3: The mobile node move towards the particular destination node D in a hop-by-hop manner.

Step 4: Source node checks the packet-dropping ratio towards the destination. If the PDR is more, it will select the alternative route towards the particular destination.

Step 5: In each pause time, the node verifies the channel activity of the particular route.

Step 6: Destination node determines the overall congestion standard value of the particular route.

Step 7: Destination sends the overall congestion standard value and sends it to the source node.

Step 8: Finally the source node sends the data through the path with minimum overall congestion standard and packet dropping ratio.

Step 9: Once the route discovery and route maintenance is established, the routing table of the all the nodes in the particular route is updated.

\section{Proposed Packet Format}

\begin{tabular}{|l|l|l|l|l|l|l|}
\hline $\begin{array}{l}\text { Source } \\
\text { ID }\end{array}$ & $\begin{array}{l}\text { Dest. } \\
\text { ID }\end{array}$ & $\begin{array}{l}\text { Hop } \\
\text { Count }\end{array}$ & MAC & OCS & $\begin{array}{l}\text { Queue } \\
\text { length }\end{array}$ & C $_{\text {act }}$ \\
\hline
\end{tabular}

Figure1. Proposed packet format

- The proposed packet is shown in Fig.1. It contains source id, destination id occupies 2 bytes. Source id is used to identify the node, which is ready to discover the route. Destination node verifies the packet received from route, which contains source id.

- The Hop count is incremented once the packet is successfully sent. It occupies 1 byte field. Hop count determines number of nodes is connected to the particular node.

- Medium Access Control (MAC) is for accessing the particular channel.

- Overall Congestion Standard is used to verify the congestion aware route. It occupies 4-byte field.

- Queue length occupies 2 bytes field, which verifies the packet dropping ratio.

- Channel activity $\mathrm{C}_{\text {act }}$ is used to verify the occupation of the channel, which occupies 2 bytes field.

\section{PERFORMANCE ANALYSIS}

We use Network Simulator (NS 2.34) to simulate our proposed algorithm. In this simulation tool, the $\mathrm{C}++$ language is back end language and tool command language (tcl) is front-end language. The basic advantage of this tool is more updation. In our simulation, 200 mobile nodes move in a 1200 meter x 1200 meter square region for 70 seconds simulation time. All nodes have the same transmission range of 250 meters. The simulated traffic is Constant Bit Rate (CBR). Our simulation settings and parameters are summarized in table 1.

Table1. Simulation settings and parameters

\begin{tabular}{|l|l|}
\hline No. of Nodes & 200 \\
\hline Area Size & 1200 X 1200 \\
\hline Mac & 802.11 \\
\hline Radio Range & $250 \mathrm{~m}$ \\
\hline Simulation Time & $70 \mathrm{sec}$ \\
\hline Traffic Source & CBR \\
\hline Packet Size & 512 bytes \\
\hline Mobility Model & Random Way Point \\
\hline Protocol & Dynamic Source Routing \\
\hline Pause time & 5 msec \\
\hline
\end{tabular}

\section{A. Topology Used}

Fig. 2 indicates the topology creation. Fig.3 illustrates the creation of source, destination and intermediate node. Once the parent node and destination has selected, the neighbour node has to be selected for route discovery. Here nodes maintain route cache and uses destination sequence number for each route entry. Omni directional antenna is used for the transmission. Route discovery mechanism is initiated when broadcasting a route request packet needs a route to a new destination. Each node selects other child nodes. This arrangement has been arranged in a hierarchical manner. The sequence number of nodes indicated the packets received from its parent node. Each route has a destination node. 


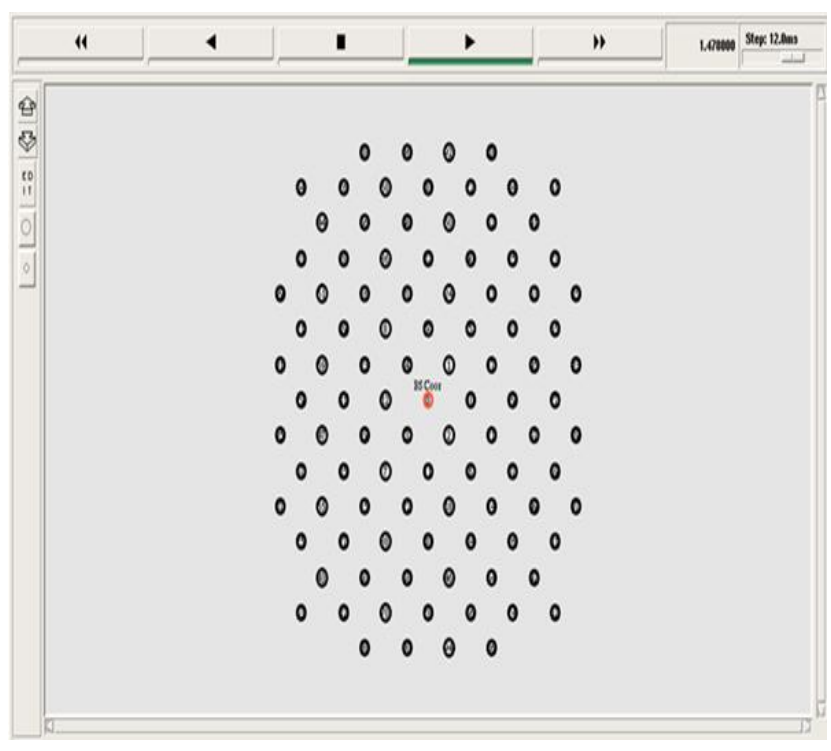

Figure 2. Topology Creation

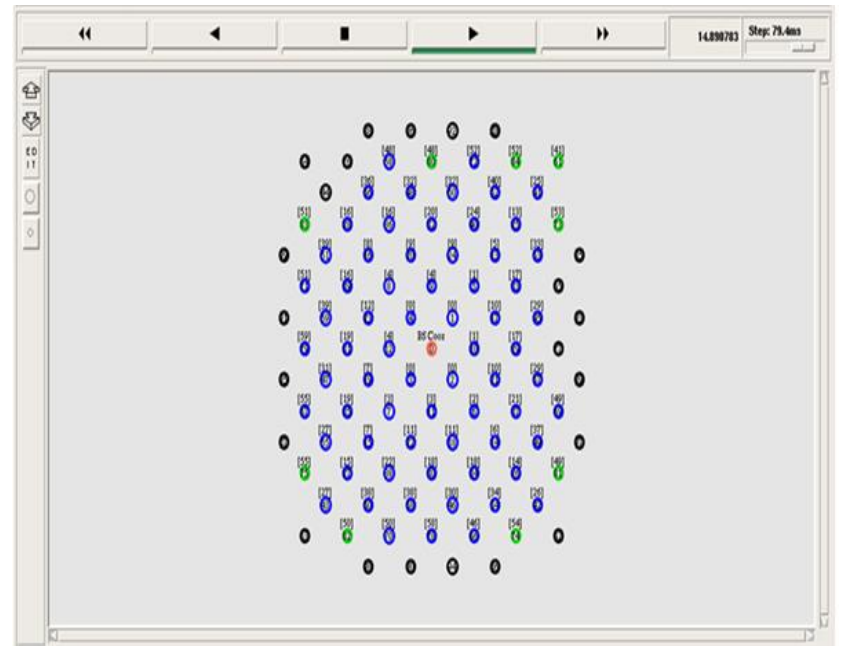

Figure 3. Source, Intermediate and Destination Node creation

\section{B. Performance Metrics}

We evaluate mainly the performance according to the following metrics.

Control overhead: The control overhead is defined as the total number of routing control packets normalized by the total number of received data packets.

End-to-end delay: The end-to-end-delay is averaged over all surviving data packets from the sources to the destinations.

Packet Delivery Ratio: It is the ratio of the number .of packets received successfully and the total number of packets transmitted.

Throughput: It is defined as the number of packet received at a particular point of time.

The simulation results are presented in the next part. We compare our ECAS with the CoDio ABCD protocol [20] in presence of congestion environment.

Fig. 4 shows the results of packet delivery ratio for varying the time from 10 to 50 . From the results, we can see that ECAS scheme has higher delivery ratio than the CoDio ABCD protocol because of congestion aware routing.

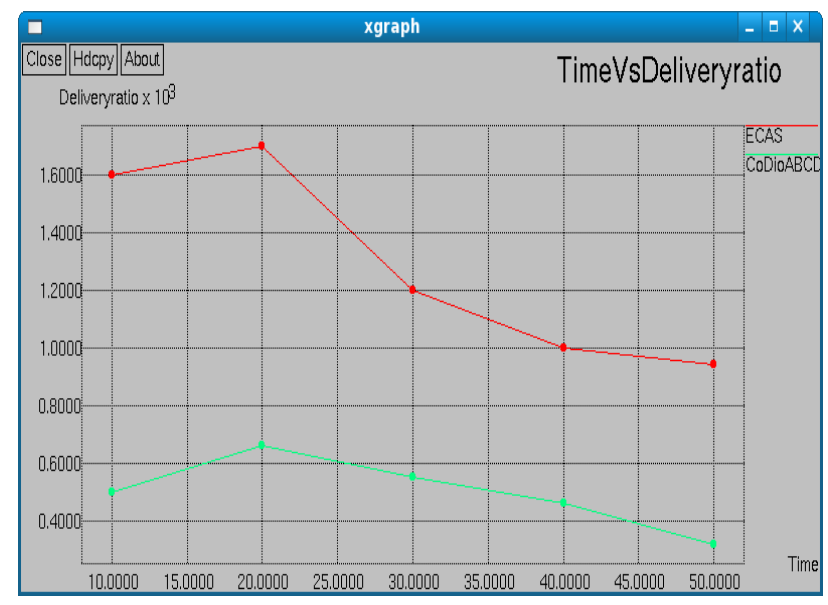

Figure 4. Time Vs Delivery Ratio

Fig. 5 shows the results of packet delivery ratio for varying the throughput from 10 to 50. From the results, we can see that ECAS scheme has higher delivery ratio than the CoDio ABCD protocol because of congestion aware routing and monitoring.

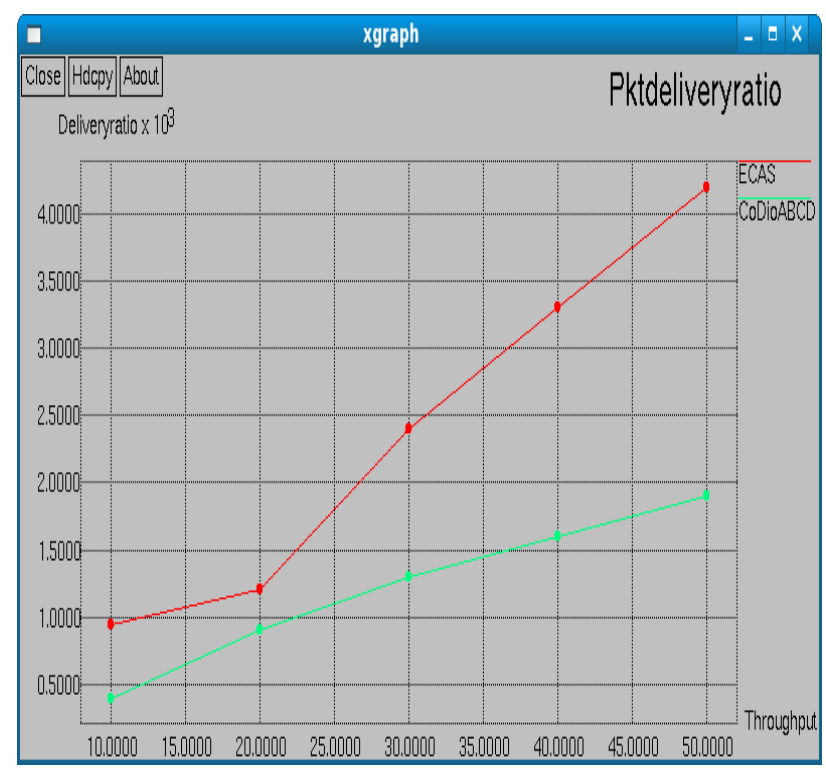

Figure 5. Throughput Vs delivery ratio

Fig. 6, presents the comparison of overhead and mobility. It is clearly shown that the overhead of ECAS has low overhead than the CoDio ABCD protocol. 


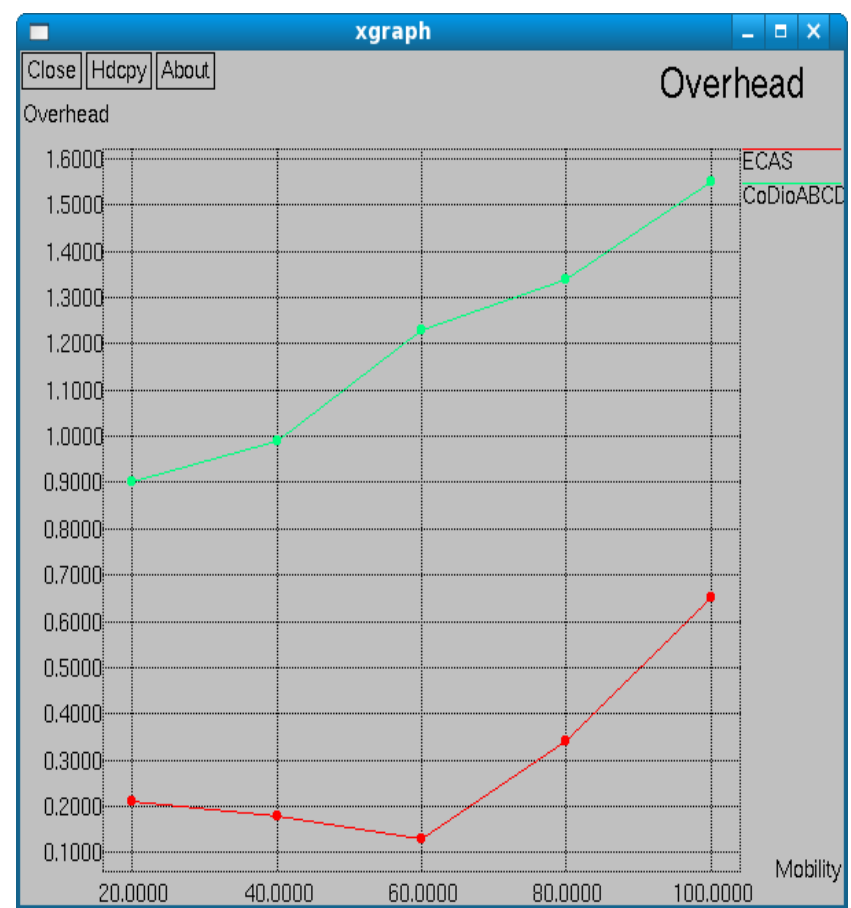

Figure 6. Mobility Vs Overhead

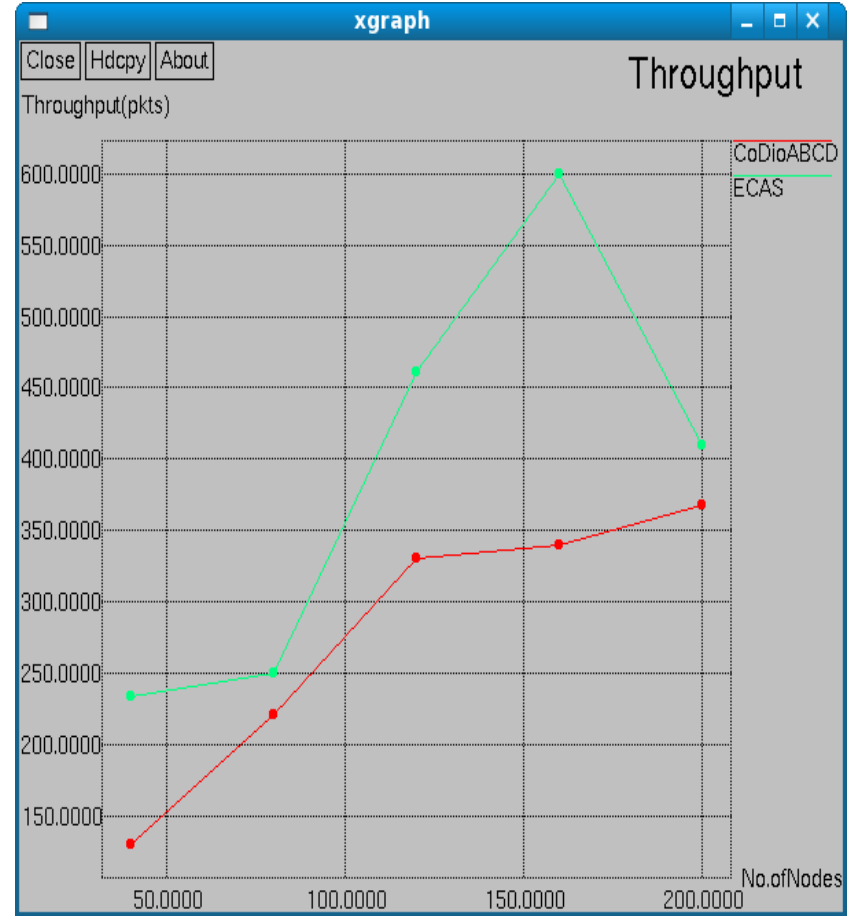

Figure 7. No.of nodes Vs Throughput

Fig. 7 shows the results of No. of Nodes Vs Throughput. From the results, we can see that ECAS scheme has higher throughput than the CoDio ABCD protocol while varying the number of nodes from 10 to 200.

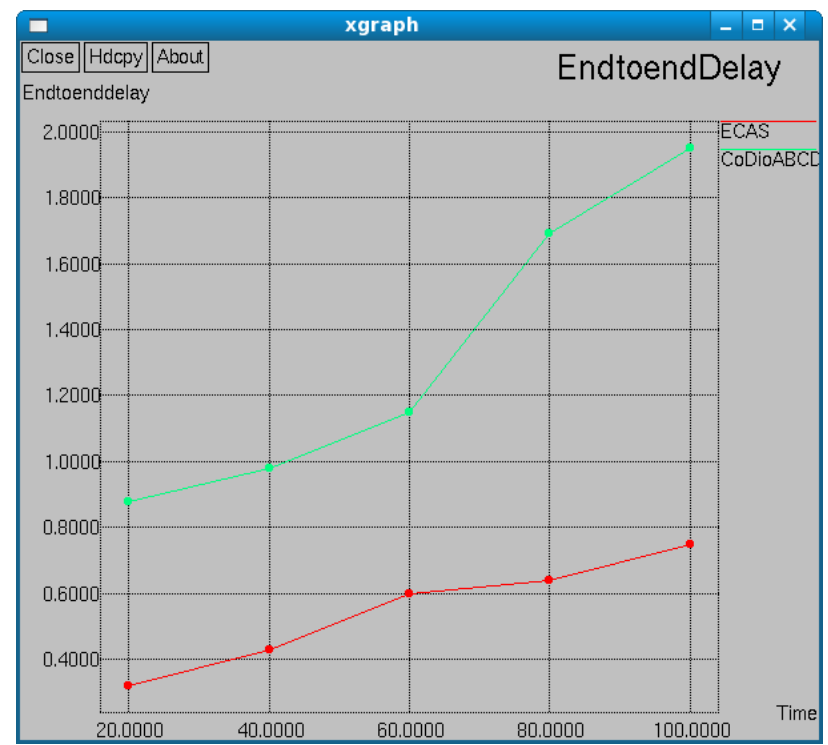

Figure 8. Time Vs Delay

Fig. 8, presents the comparison of delay while varying the time from 20 to 100 . It is clearly shown that the delay of ECAS has low than the CoDio ABCD protocol.

\section{CONCLUSION}

Mobile nodes are moving randomly without any centralized administration in MANETs. Due to high mobility the packet loss occurs unnecessarily. In this paper, we have developed a mobility based congestion control scheme, which attains congestion control and flow control among nodes. In the first phase of the scheme, congestion adaptive monitoring is achieved. Here the congestion status is totally monitored by the packet buffered to buffer size. In second phase, congestion detection is proposed. It uses the three factors like queue length, channel contention and overall congestion standard. In third phase, the congestion less routing is proposed to select the path from source to destination node through the overall congestion standard. By using the extensive simulation results, the proposed scheme ECAS achieves the better throughput, packet delivery ratio, low delay and overhead than the existing schemes while varying the mobility, time and number of nodes.

\section{REFERENCES}

[1] Prof. S.A. Jain, Mr. Abhishek Bande, Mr. Gaurav Deshmukh, Mr. Yogesh Rade and Mr. Mahesh Sandhanshiv, "An Improvement In Congestion Control Using Multipath Routing In Manet", International Journal of Engineering Research and Applications (IJERA), Vol. 2, Issue 3, May-Jun 2012, pp. 509-514. 
[2] Khuzairi Mohd Zaini, Adib M. Monzer Habbal, Fazli Azzali, Suhaidi Hassan and Mohamad Rizal, "An Interaction between Congestion-Control Based Transport Protocols and Manet Routing Protocols", Journal of Computer Science, Vol.8 (4, 2012), pp.468-473.

[3] V. Thilagavathe and Dr. K. Duraiswamy, "Cross Layer based Congestion Control Technique for Reliable and Energy Aware Routing in MANET", International Journal of Computer Applications (0975 - 8887), Volume 36- No.12, December 2011, pp.1-6.

[4] S.Karunakaran \& P.Thangaraj, "A Cluster Based Congestion Control Protocol For Mobile Ad hoc Networks", International Journal of Information Technology and Knowledge Management, JulyDecember 2010, Volume 2, No. 2, pp. 471-474.

[5] S.A.Jain and Sujata K.Tapkir, "A Review of Improvement in TCP congestion Control Using Route Failure Detection in MANET", Network and Complex Systems, Vol 2, No.2, 2012, pp.9-13.

[6] Senthil Kumaran, T. and V. Sankaranarayanan, "Congestion Free Routing in Adhoc Networks", Journal of Computer Science, Vol.8 (6), 2012, pp.971-977.

[7] S.Rajeswari, Dr.Y.Venkataramani, “Congestion Control and QOS Improvement for AEERG protocol in MANET "International Journal on AdHoc Networking Systems (IJANS) Vol. 2, No. 1, January 2012, pp.13-21.

[8] Rajkumar, G. and K. Duraiswamy, “A Fault Tolerant Congestion Aware Routing Protocol for Mobile Adhoc Networks", Journal of Computer Science, Vol.8 (5), 2012, pp.673-680.

[9] T.Suryaprakash Reddy and Dr.P.Chenna Reddy, "OCC: Ordered congestion control with cross layer support in Manet routing", International Journal of Advanced Computer Science and Applications, Vol. 3, No. 3, 2012, pp.94-101.

[10] S.A.Jain, S.R.Kokate, Pranita Thakur, Shubhangi Takalkar, "A Study of Congestion Aware Adaptive Routing Protocols in MANET", International Journal of Scientific and Research Publications, Volume 2, Issue 4, April 2012, pp.1-5.

[11] D.Revathi, K.Manikandan \& M.A.Saleemdurai, "Framework for Congestion Control with Efficient Energy and Qos Enhancement for Wireless Network", International Journal of Computer Applications (0975 - 8887), Volume 42- No.14, March 2012, pp.45-51.

[12] Prof. Shitalkumar Jain, Miss.Sunita I. Usturge, "Signal Strength Based Congestion Control in MANET", Advances in Physics Theories and Applications, Vol 1, 2011, pp.26-36.
[13] Mohammad Amin Kheirandish Fard, Sasan Karamizadeh, Mohammad Aflaki, "Enhancing Congestion Control To Address Link Failure Loss over Mobile Ad-Hoc Network", International Journal of Computer Networks \& Communications (IJCNC) Vol.3, No.5, Sep 2011,pp.177-192.

[14] Kazuya Nishimura and Kazuko Takahashi, "A Multi-Agent Routing Protocol with Congestion Control for MANET", European Conference on Modelling and Simulation, 2007, pp.1-6.

[15] Nazia Zaman, Kazi Chandrima Rahman and Syed Faisal Hasan, "Explicit Rate-based Congestion Control for Multimedia Streaming over Mobile Ad hoc Networks", International Journal of Electrical \& Computer Sciences IJECS-IJENS Vol: 10, No. 04, 2010, pp.28-40.

[16] Chen, X., Jones, H.M., Jayalath, A.D.S. Congestion-Aware Routing Protocol for Mobile Ad Hoc Networks. IEEE $66^{\text {th }}$ Conference in Vehicular Technology, 2007.

[17] Lochert, C., Scheuermann, B., Mauve, "A Survey on Congestion Control for Mobile Ad-Hoc Networks". Wireless Communications and Mobile Computing, InterScience, 2007.

[18] http://www.linktionary.com/c/congestion.html

[19] Tran, D.A., Raghavendra, H.: Congestion Adaptive Routing in Mobile Ad Hoc Networks. IEEE Transactions on Parallel and Distributed Systems, 2006.

[20] Claudio Greco, Marco Cagnazzo and Béatrice Pesquet-Popescu, "Low-Latency Video Streaming With Congestion Control in Mobile Ad-Hoc Networks", IEEE Transactions On Multimedia, Vol. 14, No. 4, August 2012, pp.1337-1350.

[21] S.Santhosh Baboo, B.Narasimhan, “A Hop-by-Hop Congestion-Aware Routing Protocol for Heterogeneous Mobile Ad-hoc Networks", International Journal of Computer Science and Information Security, Volume 3- No.1, 2009.

S.Sheeja, Born in Palakkad, Kerala state in India, in 1979, received B.Sc in Electronics from V.L.B Janaki Ammal College of Arts and Science, Coimbatore, MCA degree from Bharathiar University, M.Phil degree in Computer Science from Bharathiar University, Coimbatore in 2000 , 2003 and 2007 respectively. She has 9 years of teaching experience. She is currently pursuing doctoral research in Computer Networks in Bharathiar University. Her primary research interests are related to Computer Networks. Currently, she is working as Assist Professor in Computer Applications Department at Karpagam University, Coimbatore. 
Dr.Ramachandra.V.Pujeri, Born in Bijapur, Karnataka state in India, in 1973, received the B E in Electronics and Communication Engineering from Karnataka University, Dharwad, ME in Computer Science and Engg from PSG College of Technology, Coimbatore, Ph.D in Information and Communication Engineering from Anna University, Chennai, MBA in Human Resource Management, from Pondicherry University, Pondicherry, in 1996, 2002, 2007 and 2008 respectively. He is active life member of ISTE, SSI, MIE, ACS and IEE. His has written three textbooks. He is having around 18 years of teaching experience in the various top ten engineering colleges in India. $\mathrm{He}$ is an active expert committee member of AICTE, NBA, DoEACC, NACC and various Universities in India. Currently, under him ten research scholars pursuing their Ph.D. His research interests lie in the areas of Computer Networking, Operating System, Software Engineering, Software Reliability, Modeling and Simulation, Quality of Services and Data Mining. Currently, he is working as Vice-Principal of KGiSL Institute of Technology, Coimbatore. 